Trauma Berufskrankh 2016 · 18 (Suppl 5):S506-S510 DOI 10.1007/s10039-016-0156-3

Online publiziert: 30 . Mai 2016

(c) Springer-Verlag Berlin Heidelberg 2016

CrossMark

J. Gille · A. Paech · C. Jürgens

Klinik für Orthopädie und Unfallchirurgie, Universitätsklinikum Schleswig-Holstein, Campus Lübeck, Lübeck, Deutschland

\title{
Operative und konservative Therapie der vorderen Kreuzbandruptur im Sport
}

sprechend einer Inzidenz von 34 Operationen pro 100.000 Bürger bzw. 85 pro 100.000 Bürger in der Risikoaltersgruppe von 16 bis 39 Jahren [3]. In den USA sind jährlich etwa 120.000 Athleten von Verletzungen der Kreuzbänder betroffen [4].

Hinsichtlich der prozentualen Anteile von VKB-Verletzungen an der Gesamtzahl aller Verletzungen dominieren weibliche Sportlerinnen die Auflistung in den Sportarten Fußball, Lacrosse, Turnen und Basketball. Bezogen auf die VKBVerletzungsrate pro $1000 \mathrm{~h}$ Sport gilt Gymnastik für Frauen als gefährlichste Sportart, dicht gefolgt von American Football, weiblichem Fußball und Basketball [5].

Den Fußball betreffend, zeigen die Daten, dass weibliche Spielerinnen ein 6-fach höheres VKB-Verletzungsrisiko im Vergleich zu den männlichen Spielern besitzen. Die berichtete Inzidenz für VKB-Verletzungen reicht von 0,06-3,7 pro $1000 \mathrm{~h}$ aktiver Spielzeit in Training und Wettkampf [5]. Nach einer Umfrage in Norwegen auf Grundlage chirurgischer Aufzeichnungen wird eine jährliche Häufigkeit von VKB-Verletzungen von $0,5-6,0 \%$ bei weiblichen Spielerinnen und von $0,6-8,5 \%$ bei männlichen Spielern berichtet [2]. Die verletzten Spielerinnen waren signifikant jünger (19 vs. 23 Jahre) als ihre männlichen Kollegen [6].

\section{Diagnostik}

Die Anamnese ist auch im Zeitalter hoch technisierter bildgebender Medizin Ba- sis aller weitergehenden Diagnostik. Bereits die Anamneseerhebung kann wichtige Informationen liefern, die auf die Art und Schwere der Knieverletzung schließen lassen.

Die klinische Untersuchung gliedert sich in Inspektion, Palpation, Funktionsprüfung, Stabilitätsprüfung und spezifische Untersuchung auf Differenzialdiagnosen.

Aufgrund der besonderen Häufigkeit kommt der Funktionsprüfung des VKB eine besondere Bedeutung zu.

Zur Stabilitätstestung des VKB haben sich in der täglichen Praxis 3 Untersuchungsmethoden etabliert:

- der Lachman-Test,

- der Pivot-shift-Test und

- der vordere Schubladentest.

Der Pivot-shift-Test besteht aus einer provozierten Subluxation des Schienbeins nach innen. Der Untersucher drückt den Unterschenkel mit der einen Hand beim liegenden Patienten in Richtung Knie und führt gleichzeitig eine Innenrotation aus. Mit der anderen Hand beugt er das Knie und setzt es unter Valgusstress. Der Test zeichnet sich durch eine hohe Spezifität und eine geringe Sensitivität aus [7].

Der Lachman-Test ist ein Schubladentest, bei dem das zu testende Knie in ca. $20-30^{\circ}$ Beugung gehalten wird. Der Untersuchende umfasst den Unterschenkel mit beiden Händen so, dass die Zeigefinger in der Kniekehle liegen. Der Unterschenkel wird nach ventral gezogen. Der Lachman-Test ist ein valider Test zur De- 


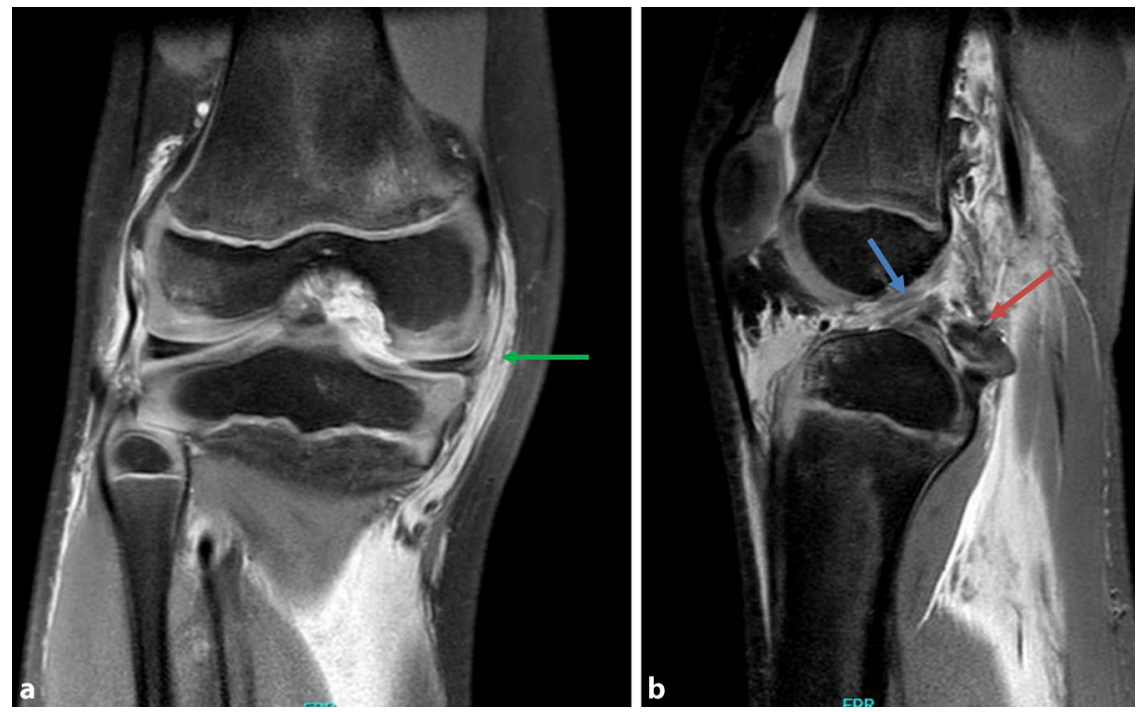

Abb. 1 ॥ MRT-Aufnahmen (T2-Wichtung) eines Kniegelenkes (9-jähriger Junge, Zustand nach Verdrehtrauma des Kniegelenkes auf einem Spielplatz). a Ruptur des medialen Kollateralbandes (grüner Pfeil). b Ruptur des vorderen (blauer Pfeil) sowie des hinteren (roterPfeil) Kreuzbandes

tektion von Kreuzbandverletzungen mit einer ausgezeichneten Sensitivität [8].

Beim Schubladentest wird das gebeugte Knie vom Untersucher mit beiden Händen so umfasst, dass die Zeigefinger beider Hände in der Kniekehle des Patienten liegen. Der Unterschenkel wird nun nach vorn gezogen oder nach hinten gedrückt. Beim vorderen Schubladentest wird der Unterschenkel gegen den Oberschenkel ventral und beim hinteren Schubladentest entsprechend dorsal verschoben. Das Ausmaß der dabei möglichen Parallelverschiebung - in Relation zum nicht betroffenen Knie - ist zusammen mit der Qualität des Endpunktes der Verschiebung wichtig für die Beurteilung [9]. Der Schubladentest zeigt in der Literatur die geringste Sensitivität der oben genannten Untersuchungsmethoden [10].

Das aktuell publizierte "lever sign“ soll die diagnostische Lücke der Partialrupturen schließen. Während die oben genannten Untersuchungen hier nur eine geringe Sensitivität aufwiesen, lag die Sensitivität für das "lever sign“ bei $100 \%$ [9].

In der Primärdiagnostik nach Verletzungen des Kniegelenkes spielen nativradiologische Aufnahmen eine wichtige Rolle zum Ausschluss von Frakturen, zur Beurteilung der Gelenkstellung und zur Detektion von degenerativen Veränderungen. Auf Röntgenaufnahmen nach
A. frühe VKB-Rekonstruktion innerhalb von 10 Wochen und Physiotherapie,

B. konservative Therapie mit Physiotherapie und spätere VKB-Rekonstruktion bei Bedarf.

Innerhalb der ersten 2 Jahre unterzogen sich 23 von 59 Patienten (39\%) der Gruppe $B$ einer verzögerten VKB-Rekonstruktion, nach 5 Jahren sogar 30 Patienten (51\%). Die Autoren der Studie folgern daraus, dass die beiden Therapiegruppen äquivalent sind, und ermuntern dazu, vermehrt eine konservative Therapie anzustreben mit optionaler späterer VKBRekonstruktion. Man kann jedoch auch folgern, dass die nichtoperative Therapie eine Versagensrate von $51 \%$ aufweist. In vielen Fällen ist unter einer initial konservativen Therapie eine VKB-Rekonstruktion notwendig geworden. Hieraus ergibt sich, dass sich die Gesamtrehabilitationszeit bei initial konservativer Therapie mit verzögerter VKB-Rekonstruktion entsprechend verlängert, was für Sportler eine lange Zeit von Sportunfähigkeit bedeutet.

Die Mehrzahl der sportlich aktiven Patienten mit einer VKB-Verletzung entscheidet sich somit für eine frühzeitige VKB-Rekonstruktion. Die - Abb. 3 zeigt das arthroskopische Bild einer durchgeführten VKB-Ersatzplastik. Die Gründe dafür konnten Swirtun et al. [15] in einer prospektiven Studie mit einem Verlaufszeitraum von 2 Jahren an 73 Patienten mit frischer VKB-Ruptur, die sich hinsichtlich der Behandlungsform (konservativ/operativ) noch unschlüssig waren, herausarbeiten. Ein Grund für eine frühzeitige Entscheidung zu einer VKBRekonstruktion war bei $75 \%$ dieser Patienten, dass generell das Auftreten von Gelenkproblemen erwartet wurde, obwohl diese nicht bestanden. Eine späte Entscheidung zur Rekonstruktion wurde von $88 \%$ dieser Patientengruppe durch bisherige negative Erfahrungen mit ihrer Kniefunktion begründet.

Wünschenswert wäre deshalb, wenn man beim initialen Therapieassessment die Faktoren kennen würde, ob eine konservative Therapie erfolgreich sein wird oder nicht. Dass eine konservative Therapie erfolgreich sein kann, schildert der 
Fallbericht eines Fußballprofis aus der englischen Premier League, der 8 Wochen nach VKB-Ruptur wieder in den Sport zurückkehrte und bei der Nachuntersuchung nach 18 Monaten vollständig beschwerdefrei war [16]. In einer aktuellen Untersuchung an 2304 Fällen von VKB-Ruptur wurden etwa drei Viertel der Fälle erfolgreich konservativ therapiert. Eine VKB-Rekonstruktion war lediglich bei $22,6 \%$ der Fälle erforderlich. Bei der Analyse zeigte sich, dass die Wahrscheinlichkeit einer erforderlichen Operation bei jungen Männern mit hohem sportlichem Anspruch und gehobenem sozioökonomischem Status besonders hoch war [17].

\section{„Return to play“}

Weniger als die Hälfte der Athleten können innerhalb des ersten Jahres nach VKB-Ersatzplastik in den Sport zurückkehren. In einer aktuellen Untersuchung zeigt sich, dass nach Rückkehr der Athleten in den Sport ein Viertel eine erneute Kreuzbandverletzung erleiden wird (• Abb. 4; [18]). Die Ergebnisse nach Revisionskreuzbandchirurgie sind erwartungsgemäß schlechter als nach Erstoperation [19, 20].

Rodriguez-Roiz et al. [21] berichten, dass von 99 Patienten nach VKB-Rekonstruktion 91,9\% wieder Sport treiben konnten, wobei in $75 \%$ der Fälle dies innerhalb des ersten postoperativen Jahres möglich war. Interessanterweise konnten jedoch nur 51 Patienten (51,5\%) wieder auf dem angestammten Niveau Sport ausüben, und 24 Patienten mussten die Sportart wechseln.

Ardern et al. [22] zeigten in einer Metaanalyse, dass in $81 \%$ der Fälle nach VKB-Rekonstruktion sportliche Aktivitäten wieder aufgenommen werden konnten. Allerdings konnten nur $65 \%$ auf das vor dem Unfall bestehende Leistungsniveau zurückkehren, und nur $55 \%$ konnten auf Wettkampfniveau Sport treiben. Die Autoren vermuten, dass Athleten trotz regelhaftem Heilverlauf nach Kreuzbandverletzung sportliche Prioritäten anders gesetzt haben könnten und bei subjektiver Zufriedenheit auf einem niedrigeren Niveau Sport treiben. Für eine erfolgreiche Wiederauf-

Trauma Berufskrankh 2016 · 18 (Suppl 5):S506-S510 DOI 10.1007/s10039-016-0156-3

(c) Springer-Verlag Berlin Heidelberg 2016

\section{J. Gille · A. Paech · C. Jürgens \\ Operative und konservative Therapie der vorderen Kreuzbandruptur im Sport}

\section{Zusammenfassung}

Verletzungen der Kreuzbänder sind im Sport häufig und zählen zu den häufigsten Ursachen für Sportunfähigkeit. Bei der vorderen Kreuzband (VKB)-Ruptur sind konservative und operative Therapieoptionen zu diskutieren. Wie sind hierzu die aktuellen Therapieempfehlungen? Die vorliegende Arbeit greift genau diese Fragestellung auf und stellt neben den diagnostischen Methoden die operativen und konservativen Therapieoptionen dar. In der Diagnostik steht die Anamneseerhebung an erster Stelle. Zur Stabilitätsprüfung des VKB sind folgende, klinische Tests etabliert: der LachmanTest, der Pivot-shift-Test und der vordere Schubladentest. Während das Nativröntgen knöcherne Begleitverletzungen detektieren kann, ist die Magnetresonanztomographie zur Diagnostik der Kreuzbandverletzung geeignet. Während die konservative Therapie der VKB-Läsion erfolgreich sein kann, zeigt sich, dass v. a. bei hohem sportlichem Anspruch eine operative VKB-Rekonstruktion häufiger erforderlich wird. Dennoch kann durch eine VKB-Rekonstruktion die ursprüngliche Kniefunktion nicht immer identisch wiederhergestellt werden. So ist zu erklären, dass nicht alle Sportler auf das vor der Kreuzbandläsion bestehende Leistungsniveau zurückkehren können und nur $55 \%$ auf Wettkampfniveau Sport treiben können.

Schlüsselwörter

Knie · Verletzung · Anamnese · Magnetresonanztomographie $\cdot$ Röntgen

\section{Surgical and conservative treatment of anterior cruciate ligament rupture in sports}

\section{Abstract}

Injuries of the cruciate ligaments are frequent in sport and are one of the most frequent causes of sport invalidity. Conservative and surgical treatment options are possible for ruptures of the anterior cruciate ligament. What are the current treatment recommendations? This article examines exactly this question and presents the surgical and conservative therapy options. The anamnesis is given priority in the diagnostics. The following are established tests to investigate the integrity of the anterior cruciate ligament: the Lachman test, the pivot shift test and the anterior drawer test. Whereas accompanying bony injuries can be detected by native X-ray imaging, magnetic resonance imaging (MRI) is suitable for the diagnostics of cruciate ligament injuries. Although conservative therapy of anterior cruciate ligament lesions can be successful, it has been shown that surgical reconstruction is more often necessary, particularly for highly demanding sporting activities; however, the original knee function can never be restored in an identical fashion by reconstruction of the anterior cruciate ligament. This explains why not all athletes can return to the same performance level achieved before the cruciate ligament lesion and only $55 \%$ can maintain sport at a competition level.

\section{Keywords}

Knee $\cdot$ Injury $\cdot$ Medical history taking . Magnetic resonance imaging $\cdot \mathrm{X}$-rays nahme des Sports sprechen ein jüngeres Lebensalter, das männliche Geschlecht sowie sportliche Aktivitäten auf Leistungsniveau vor dem Unfall [22]. Als weitere Faktoren für ein erfolgreiches „return-to-play“ werden eine adäquate Kraft der Quadrizepsmuskulatur, ein geringer Reizzustand des Kniegelenkes, stabile Bandverhältnisse und ein hoher Grad an Motivation diskutiert [23].
Angst vor einer erneuten Verletzung, einer erneuten Rehabilitationsphase, aber auch vor einem Einkommensverlust im Beruf waren wesentliche Faktoren, nicht wieder auf angestammtem Niveau Sport zu treiben [24].

Bei einer Befragung im American Football gaben die verantwortlichen Teamärzte an, dass Sport 6 Monate (55,8\%) bzw. 9 Monate (12,3\%) nach 


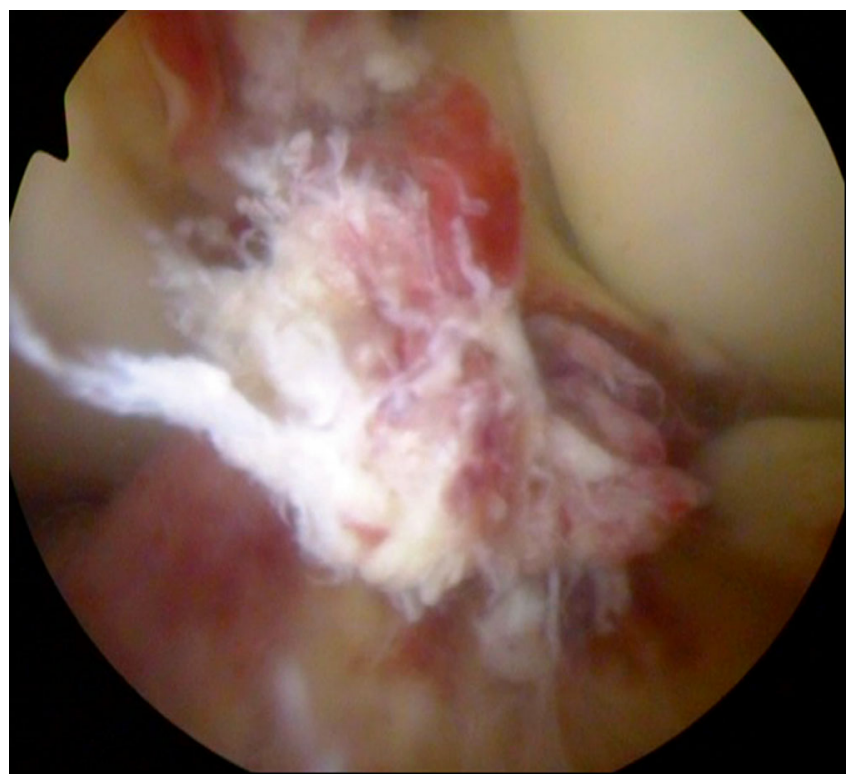

Abb. $2 \Delta$ Arthroskopisches Bild einer frischen, intraligamentären, vorderen Kreuzbandruptur

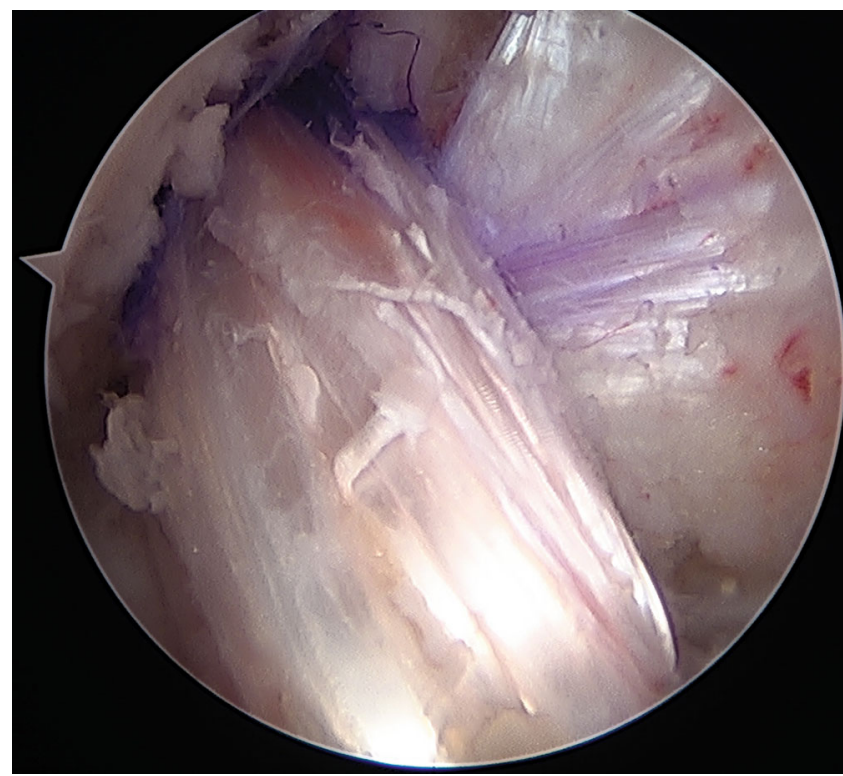

Abb. 3 A Arthroskopisches Bild nach vorderer Kreuzbandersatzplastik mit einem 4-fach gelegten Semitendinosustransplantat

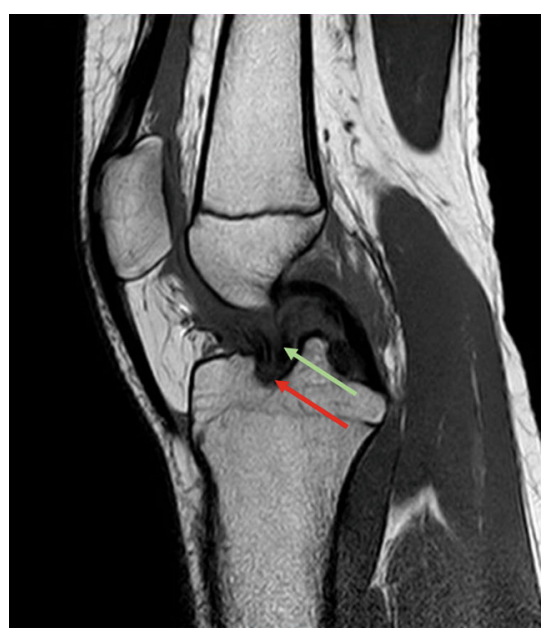

Abb. 4 \ MRT-Aufnahmen (T1-Wichtung) eines Kniegelenkes. Das Bild zeigt eine Re-Ruptur nach Rekonstruktion des vorderen Kreuzbandes (grüner Pfeil) nach erneutem Knieverdrehtrauma.Der rotePfeilzeigt auf den tibialen Bohrkanal

VKB-Rekonstruktion wieder möglich sei [25]. In keinem Fall wurde empfohlen, länger als 12 Monate zu warten. Diese Angaben decken sich mit einer aktuellen Befragung von 123 Sportärzten, von denen $87 \%$ sportliche Aktivitäten 6 Monate nach Kreuzbandoperation erlauben [26]. Die Freigabe für Kontaktsportarten erteilen zu diesem Zeitpunkt allerdings nur $53 \%$.
$\mathrm{Ob}$ eine VKB-Rekonstruktion einer Degeneration des Kniegelenkes vorbeugen kann, bleibt unklar. Gesichert ist, dass bei Verletzung des VKB Begleitpathologien wie u. a. Meniskusrisse, „bone bruise" des angrenzenden Knochens sowie ein persistierender Gelenkerguss mit der Entstehung von degenerativen Veränderungen assoziiert sind [27]. Ob anterior cruciate ligament(ACL)-Verletzungen konservativ oder operativ behandelt werden, scheint hingegen keinen Einfluss auf die Entstehung einer Gonarthrose zu haben. So zeigte sich in einer aktuellen Untersuchung 10 Jahre nach VKB-Ruptur, dass die Entstehung einer Gonarthrose durch die operative Versorgung mittels Hamstring-Sehnentransplantat im Vergleich zur konservativen Therapie nicht verhindert werden kann [28].

\section{Fazit für die Praxis}

\section{- Die Struktur des VKB ist komplex.}

- Deshalb scheint es gerade in diesem Zusammenhang wichtig, die betroffenen Athleten, Trainer und das Athletenumfeld darüber aufzuklären, dass durch eine VKB-Rekonstruktion die ursprüngliche Kniefunktion häufig nicht identisch wiederhergestellt werden kann.

\section{Korrespondenzadresse}

\section{PD. Dr. med. J. Gille}

Klinik für Orthopädie und Unfallchirurgie, Universitätsklinikum Schleswig-Holstein, Campus Lübeck

Ratzeburger Allee 160, 23538 Lübeck, Deutschland

justus.gille@usa.net

\section{Einhaltung ethischer Richtlinien}

Interessenkonflikt. J. Gille, A. Paech und C. Jürgens geben an, dass kein Interessenkonflikt besteht.

Dieser Beitrag beinhaltet keine von den Autoren durchgeführten Studien an Menschen oder Tieren.

The supplement containing this article is not sponsored by industry.

\section{Literatur}

1. Ahern BJ et al (2009) Preclinical animal models in single site cartilage defect testing: a systematic review. Osteoarthr Cartil 17(6):705-13. doi:10. 1016/j.joca.2008.11.008

2. Renstrom $P$ et al (2008) Non-contact $A C L$ injuries in female athletes: an International Olympic Committee current concepts statement. BrJSports Med 42(6):394-412

3. Granan LP et al (2009) The Scandinavian ACL registries 2004-2007: baseline epidemiology. Acta Orthop 80(5):563-567

4. Majewski M, Susanne H, Klaus S (2006) Epidemiology of athletic knee injuries: A 10-year study. Knee 13(3):184-188 
5. Stanley LE et al (2016) Sex differences in the incidence of anterior cruciate ligament, medial collateral ligament, and meniscal injuries in collegiate and high school sports: 2009-2010 through 2013-2014. Am J Sports Med. doi:10.1177/0363546516630927

6. Walden Metal (2011) Theepidemiology of anterior cruciate ligament injury in football (soccer): a review of the literature from a gender-related perspective. Knee Surg Sports Traumatol Arthrosc 19(1):3-10

7. Huang W et al (2016) Clinical examination of anterior cruciate ligament rupture: a systematic review and meta-analysis. Acta Orthop Traumatol Turc 50(1):22-31

8. Stanev D et al (2016) ACL reconstruction decision support. personalized simulation of the Lachman test and custom activities. Methods Inf Med 55(1):98-105

9. Lelli A et al (2014) The "Lever Sign": a new clinical test for the diagnosis of anterior cruciate ligament rupture. Knee Surg Sports Traumatol Arthrosc. doi:10.1007/s00167-014-3490-7

10. Leblanc MC et al (2015) Diagnostic accuracy of physical examination for anterior knee instability: a systematic review. Knee Surg Sports Traumatol Arthrosc 23(10):2805-2813

11. Werner BC et al (2016) Limited utility of routine early postoperative radiography after primary $A C L$ reconstruction. Knee 23(2):237-240

12. Dufka FL et al (2016) Accuracy of MRI evaluation of meniscus tears in the setting of $A C L$ injuries. Knee. doi:10.1016/j.knee.2016.01.018

13. Frobell RB et al (2010) A randomized trial of treatment for acute anterior cruciate ligament tears. NEngl J Med 363(4):331-342

14. Frobell RB et al (2013) Republished research: treatment for acute anterior cruciate ligament tear: five year outcome of randomised trial. Br J Sports Med 47(6):373

15. Swirtun LR, Eriksson K, Renstrom P (2006) Who chooses anterior cruciate ligament reconstruction and why? A 2-year prospective study. Scand J Med Sci Sports 16(6):441-446

16. Weiler R et al (2015) Non-operative management of a complete anterior cruciate ligament injury in an English Premier League football player with return to play in less than 8 weeks: applying common sense in the absence of evidence. BMJ Case Rep 2015(apr261):bcr2014208012

17. Collins JE et al (2013) Cumulative incidence of ACL reconstruction after $A C L$ injury in adults: role of age, sex, and race. Am J Sports Med 41 (3):544-549

18. Johnson WR et al (2015) Patient outcomes and predictors of success after revision anterior cruciate ligament reconstruction. Orthop J Sports Med 3(10):2325967115611660

19. Wiggins AJ et al (2016) Risk of secondary injury in younger athletes after anterior cruciate ligament reconstruction: A systematic review and meta-analysis. Am J Sports Med. doi:10.1177/0363546515621554

20. Grassi A et al (2016) Does revision ACL reconstruction measure up to primary surgery? A metaanalysis comparing patient-reported and clinicianreported outcomes, and radiographic results. $\mathrm{Br} \mathrm{J}$ Sports Med. doi:10.1136/bjsports-2015-094948

21. Rodriguez-Roiz JM et al (2015) Return to recreational sports activity after anterior cruciate ligament reconstruction: a one- to six-year follow-up study. Arch Orthop Trauma Surg 135(8):1117-1122

22. Ardern CL et al (2011) Return to sport following anterior cruciate ligament reconstruction surgery: a systematic review and meta-analysis of the state of play. Br J Sports Med 45(7):596-606

23. Czuppon S et al (2014) Variables associated with return to sportfollowing anterior cruciate ligament reconstruction: a systematic review. Br J Sports Med 48(5):356-364

24. Tjong VK et al (2014) A qualitative investigation of the decision to return to sport after anterior cruciate ligament reconstruction: to play or not to play. Am J Sports Med 42(2):336-342

25. Erickson BJ et al (2014) Anterior cruciate ligament reconstruction practice patterns by NFL and NCAA football team physicians. Arthroscopy 30(6):731-738

26. Grassi A et al (2016) Return to sport after ACL reconstruction: a survey between the Italian Society of Knee, Arthroscopy, Sport, Cartilage and Orthopaedic Technologies (SIGASCOT) members. Eur J Orthop Surg Traumatol. doi:10.1007/s00590 016-1756-0

27. Meer BL van et al (2016) Degenerative changes in the knee 2 years after anterior cruciate ligament rupture and related risk factors: A prospective observational follow-up study. Am J Sports Med. doi:10.1177/0363546516631936

28. Tsoukas D et al (2015) No difference in osteoarthritis after surgical and non-surgical treatment of $A C L$-injured knees after 10 years. Knee Surg Sports Traumatol Arthrosc. doi:10.1007/s00167015-3593-9 